\title{
An Experimental and Theoretical Analysis of Jitter in Self-Pulsating Lasers Synchronized to Periodic Electrical Signals
}

\author{
A. Egan, M. Harley-Stead, P. Rees, S. Lynch, J. O'Gorman, and J. Hegarty
}

\begin{abstract}
We investigate both experimentally and theoretically the behavior of pulse jitter in compact disk (CD) self-pulsating lasers that have been synchronized to an applied periodic electrical signal. Our investigations reveal that pulse-to-pulse jitter in a synchronized laser is almost entirely unchanged from that in an unsynchronized laser under the same bias conditions. The major effect of synchronization is the suppression of long term jitter.
\end{abstract}

\section{INTRODUCTION}

$\mathbf{I}$ T IS widely believed that self-pulsating (SP) laser diodes (LD's) will have an important role to play in optical communications. Clock extraction and the potential for optical clock distribution by synchronization of laser diode selfpulsation to applied electrical and optical signals have already been demonstrated [1], [2]. To date there has been considerable effort to develop an understanding of high speed selfpulsations in multisection Fabry-Perot and distributed feedback laser diodes operating in the long wavelength telecommunications windows around $1.3 \mu \mathrm{m}$ and $1.5 \mu \mathrm{m}$. For relatively low component volume applications such as high-speed longhaul systems, clock extraction may be performed using current commercial electronic systems at data rates up to $10 \mathrm{~Gb} / \mathrm{s}$. Consequently, a major thrust of research in this arena is in development of devices that pulsate or modelock stably at frequencies in excess of $40 \mathrm{GHz}$. At lower data rates characteristic of future high-speed datacom applications (1-2 Gb/s) there is a role for inexpensive, mass producable subsystems for clock extraction and distribution. Such clocking optical subsystems will require cheap self-pulsating laser sources, a role readily filled by $\mathrm{CD}$ laser diodes. For such subsystems, an important consideration in determining the fidelity of the clock extracted from a data stream is a knowledge of the contribution to timing jitter in the derived clock by the self-pulsating laser dynamics.

In this letter we present experimental results, obtained using a cross-correlation technique, concerning timing jitter in synchronized self-pulsating CD lasers. We compare jitter in free-running self-pulsating lasers with jitter in synchronized

Manuscript received October 12, 1995; revised February 23, 1996. This work was supported by the Commission of the European Communities under a Human Capital and Mobility Network, Contract CHRX-CT94-0594 (administered by France Telecom). One of the authors, P. Rees, was supported by the EC under the Human Capital Mobility research training programme.

The authors are with Optronics Ireland, Physics Department, Trinity College, Dublin 2, Ireland.

Publisher Item Identifier S 1041-1135(96)04299-1.

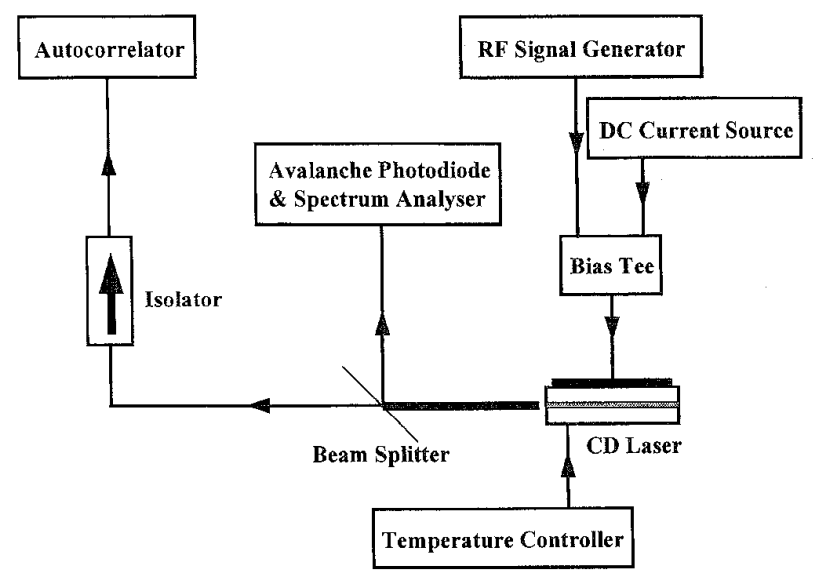

Fig. 1. Experimental set-up for the measurement of $C D$ laser diode self-pulsation characteristics. PD is a 2-GHz bandwidth Avalanche photodiode.

self-pulsating lasers. Our results give very good agreement with calculations based on a well known model for CD laser diodes. This shows that synchronization of laser diode selfpulsations to an applied electrical signal does not lead to a reduction of pulse-to-pulse timing jitter, but rather leads to prevention of jitter accumulation over a long pulse train.

Fig. 1 shows the experimental arrangement. The CD laser diode is a Sharp model LT022. A low amplitude sinusoidal electrical signal is applied to the laser, along with the normal dc-bias current, through a bias tee. The LD emission passes through a beam splitter-one portion of which is monitored with a 2.2-GHz bandwidth avalanche photodiode and RF spectrum analyzer, while the other goes through a Faraday optical isolator to an autocorrelator.

Here, we use auto- and cross-correlation methods for pulseto-pulse jitter measurement [3]. Jitter in laser pulse trains is commonly determined by measurement of high frequency harmonics in the detected optical signal RF power spectrum [4]. Such measurements however, determine average timing jitter over the entire pulse train. In this paper we use auto and cross-correlation techniques to estimate jitter since our primary purpose is to examine jitter at the pulse-to-pulse level. In autocorrelation, the time-averaged output from the second harmonic crystal depends on the time delay between the two arms, $\tau$, and is a-maximum when $\tau$ is an integer multiple of the average pulse period, $\tau_{p}$. When $\tau$ is varied 


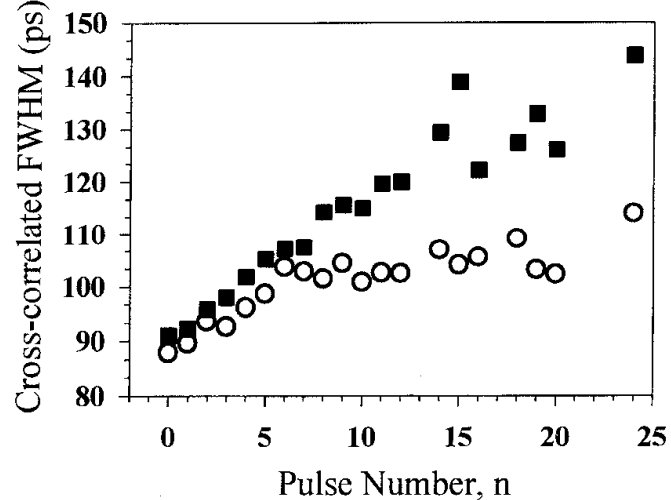

Fig. 2. Plot of measured 2-GHz CD laser cross-correlation FWHM versus pulse number, $n$. The LD free-running self-pulsation frequency in each case is approximately $2 \mathrm{GHz}$ and the laser is synchronized to an applied electrical sinusoidal signal of $2 \mathrm{GHz}$. The symbols represent the following data. (a) Squares: Experimental data for a free-running laser. (b) Circles: Experimental data for electro-optically synchronized laser.

about 0 , an autocorrelation trace is obtained whose full width at half maximum (FWHM) is equal to the average pulse width multiplied by a constant. When $\tau$ is varied about $n \tau_{p}$, where $n$ is an integer not equal to zero, cross correlation traces are obtained. The cross-correlation FWHM at a given order, $n$, is determined by both the average pulse width (as measured when $n=0$ ) and the average timing jitter between the two temporally separated sets of pulses generating the cross correlation.

In Fig. 2, we show a plot of the cross correlation FWHM versus $n$, obtained using a $C D$ laser diode when free-running with a SP frequency of $2 \mathrm{GHz}$ and, for the same laser, when synchronized to an applied low level $(-4 \mathrm{dBm})$ sinusoidal electrical signal of frequency $2 \mathrm{GHz}$. Since the average pulse width is measured when $n=0$, any increase in FWHM seen in the cross-correlation is due to increasing jitter over $n$ periods. The FWHM increase at low $n$ is very similar for the cases of the free-running and synchronized self-pulsation. The $n=0$ FWHM for the synchronised self-pulsation is 3 ps less than for the free-running self-pulsation. Simulations show the synchronizing electrical signal slightly increases the carrier density achieved prior to pulse emission and results in the shorter optical pulse [5]. At $n>7$ the two curves begin to diverge markedly. In contrast to the behavior of the freerunning laser, the curve for the synchronized laser is flatter and the cross correlation FWHM approaches a value of about 110 ps (i.e., about 20 ps greater than its minimum value) at $n=25$, the highest order cross correlation measured. These results indicate that synchronization is not a process in which pulse-to-pulse jitter is reduced, but instead jitter accumulation is reduced.

In order to clarify the effect of synchronization on jitter in self-pulsating laser diodes we have modeled SP laser diodes and calculated the pulse emission jitter. In self-pulsating CD laser diodes operating under normal bias conditions, the optical field in the central electrically pumped active region spreads out and overlaps neighboring unpumped regions that act as a distributed saturable absorber. Self-pulsation occurs for proper

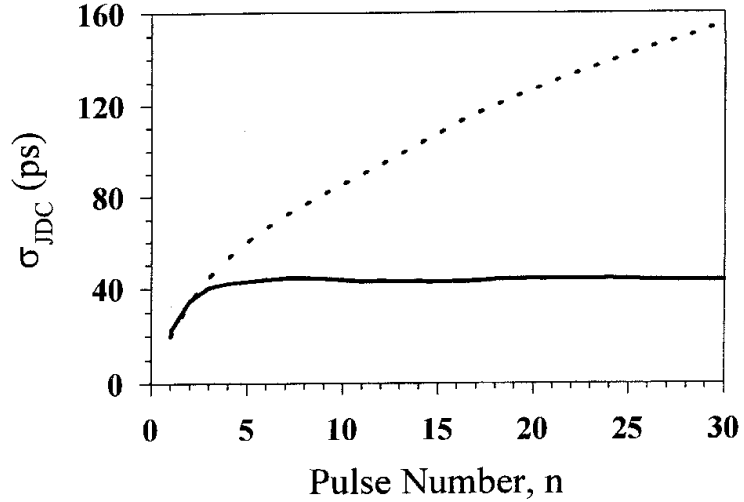

Fig. 3. $\sigma_{\mathrm{JDC}}$ versus $n$. The dotted line represents $\sigma_{\mathrm{JDC}}$ calculated for a free-running CD SP laser while the solid line represents data calculated for the $C D$ laser synchronized to a sinusoidal electrical signal

balancing of the pumped and unpumped region volumes. Yamada [6] has developed a single-mode rate equation model describing such devices that we use here to investigate selfpulsation dynamics and synchronization. In this model the photon and carrier dynamics are described using three rate equations. Two equations govern the rate of change of carrier density in the central active region and the neighboring absorbing regions respectively, while a third equation governs the rate of change of photon number in the laser cavity. We modify Yamada's model by including a spontaneous emission noise term (Langevin noise source) in the photon rate equation [7]. Noise terms in the equations for carrier density are not included since it is known that these terms are less important for fluctuation properties of the laser [7], [8]. Inclusion of spontaneous emission noise in the laser rate equations allows us to analyze the jitter properties of pulsating LD emission. In our simulations we generate 10200 laser pulses, the first 200 of which are discarded in order to discriminate against turn-on transient effects. The time between pulses separated by $n \tau_{p}$ is determined and binned to produce jitter distribution curves (JDC) whose FWHM, $\sigma_{\text {JDC }}$, measures the timing jitter between pulses separated by $n \tau_{p}$. This procedure is discussed in more detail in [9].

Fig. 3 shows the variation of $\sigma_{\text {JDC }}$ with $n$ when a CD laser is synchronized to an applied sinusoidal electrical signal and for a free-running $\mathrm{CD}$ laser. The behavior of $\sigma_{\mathrm{JDC}}$ with increasing $n$ shows the same trends as observed for the cross correlation dependence on $n$ (Fig. 2). Specifically for $n=1$ the pulse jitter for the synchronized laser and for the free-running laser are similar. $\sigma_{\text {JDC }}$ in the free-running laser increases monotonically. $\sigma_{\text {JDC }}$ for the synchronized laser saturates after about 6 pulses. The values of the FWHM and the onset of saturation of $\sigma_{\mathrm{JDC}}$ with pulse number, $n$ do not match exactly with experiment but considering the simple model used with standard laser parameters and the fact that no fitting was attempted, the agreement is good. These results clarify the observed behavior of the synchronized selfpulsating laser RF spectrum. Synchronization is characterized by a decrease in the FWHM of the fundamental peak in the emission RF power spectrum. This decrease results from a reduction in the accumulated jitter in the self-pulsating 
emission and not a decrease in pulse-to-pulse jitter. The reduction in the accumulated jitter being due to the reduced frequency drift when the periodic signal interacts with the laser. The demonstration that SP LD pulse-to-pulse jitter is not substantially changed by electrical synchronization is important since it qualifies the extent to which SP LD's may be useful in generating low-jitter optical pulse trains or as optical transmitters in RF fiber-optic links [9]. These calculations confirm that the primary effect of synchronization is to prevent an accumulation of timing jitter. These issues are also of interest where synchronization of SP LD's is achieved by injection of optical pulse trains. Despite the synchronization mechanism being slightly different, in the case of optical injection we expect similar results, this is currently under investigation.

\section{CONCLUSION}

We have made measurements of jitter in synchronized CD SP laser diodes and compared these results to measurements made for the free-running self-pulsation. We have also compared our experimental measurements with simulations of these lasers using a standard rate equation model and obtained very good agreement. Our results show that SP LD's synchronized to periodic electrical signals show almost no reduction in pulse-to-pulse jitter compared to the free-running selfpulsation, but there is a reduction in jitter accumulation. The practical implications of our results depend on the nature of the application involved. For example where synchronized SP LD's are used for optical clock extraction, long transitionless intervals in data streams may lead to pattern effects in clock timing.

\section{ACKNOWLEDGMENT}

The authors thank P. Phelan and G. Farrell for useful discussions.

\section{REFERENCES}

[1] P. E. Barnsley, G. E. Wickens, H. J. Wickes, and D. M. Spirit "All-optical clock recovery from a $5 \mathrm{~GB} / \mathrm{s} \mathrm{RZ}$ data signal using a selfpulsating $1.56 \mu \mathrm{m}$ laser diode," lEEE Photon. Technol. Lett., vol. 3, no. 10, pp. $942-945,1991$

[2] G. Farrell, P. Phelan, J. Hegarty, and J. Shields, "All-optical timing extraction with frequency division using a twin section laser diode," IEEE Photon. Technol. Lett., vol. 5, no. 6, pp. 718-721, 1993.

[3] J.-C. M. Diels, J. J. Fontaine, I. C. McMichael, and F. Simoni, "Control and measurement of ultrashort pulse shapes (in amplitude and phase) with femtosecond accuracy," Appl. Opt., vol. 24, no. 9, pp. 1270-1282, 1985.

[4] D. von der Linde "Characterization of the noise in continuously operating mode-locked lasers," Appl. Phys. B, vol. 39, pp. 201-217, 1986.

[5] A. Egan, P. Rees, J. O'Gorman, M. Harley-Stead, G. Farrell, J. Hegarty, and P. Phelan, "On the theory of electro-optical synchronization of selfpulsating laser diodes," to be published in the Feb. Edition of IEE Proc. Optoelectron., 1996.

[6] M. Yamada, "A theoretical analysis of self-sustained pulsation phenomena in narrow-stripe semiconductor lasers," IEEE J. Quantum Electron., vol. 29 , no. 5 , pp. 1330-1336, 1993.

[7] E. A. Avrutin, "Analysis of spontaneous emission and noise in selfpulsing laser diodes," IEE Proc. J., vol. 140, no. 1, pp. 16-20, 1993.

[8] G. P. Agrawal and N. K Dutta, Long Wavelength Semiconductor Lasers. New York: Van Nostrand Reinhold, 1986.

[9] J. B. Georges and K. Y. Lau, "Broadband microwave fiber-optic links with RF phase control for phased-array antennas," IEEE Photon. Technol. Lett., vol. 5, no. 11, pp. 1344-1346, 1993. 